\title{
Monocarboxylate Transporter 1 Deficiency and Ketone Utilization
}

\author{
Peter M. van Hasselt, M.D., Ph.D., Sacha Ferdinandusse, Ph.D., \\ Glen R. Monroe, M.Sc., Jos P.N. Ruiter, B.Sc., Marjolein Turkenburg, B.Sc., \\ Maartje J. Geerlings, M.Sc., Karen Duran, B.Sc., Magdalena Harakalova, M.D., Ph.D., \\ Bert van der Zwaag, Ph.D., Ardeshir A. Monavari, M.D., Ilyas Okur, M.D., Ph.D., \\ MarkJ. Sharrard, F.R.C.P.C.H., Maureen Cleary, M.D., Nuala O'Connell, M.B., Ch.B., \\ Valerie Walker, M.D., M. Estela Rubio-Gozalbo, M.D., Ph.D., Maaike C. de Vries, M.D., \\ Gepke Visser, M.D., Ph.D., Roderick H.J. Houwen, M.D., Ph.D., \\ Jasper J. van der Smagt, M.D., Nanda M. Verhoeven-Duif, Ph.D., \\ Ronald J.A. Wanders, Ph.D., and Gijs van Haaften, Ph.D.
}

\section{SUMMARY}

Ketoacidosis is a potentially lethal condition caused by the imbalance between hepatic production and extrahepatic utilization of ketone bodies. We performed exome sequencing in a patient with recurrent, severe ketoacidosis and identified a homozygous frameshift mutation in the gene encoding monocarboxylate transporter 1 (SLC16A1, also called MCT1). Genetic analysis in 96 patients suspected of having ketolytic defects yielded seven additional inactivating mutations in MCT1, both homozygous and heterozygous. Mutational status was found to be correlated with ketoacidosis severity, MCT1 protein levels, and transport capacity. Thus, MCT1 deficiency is a novel cause of profound ketoacidosis; the present work suggests that MCT1mediated ketone-body transport is needed to maintain acid-base balance.

The authors' affiliations are listed in the Appendix. Address reprint requests to Dr. van Hasselt at the Department of Metabolic Diseases, Wilhelmina Children's Hospital, UMC Utrecht, KE. 04.133.1, P.O. Box 85090, 3508 AB Utrecht, the Netherlands, or at p.vanhasselt@umcutrecht.$\mathrm{nl}$; or Dr. van Haaften at the Center for Molecular Medicine, Department of Medical Genetics, UMC Utrecht, STR. 1.305, P.O. Box 85060, 3508 AB, Utrecht, the Netherlands, or at g.vanhaaften@umcutrecht.nl.

Drs. van Hasselt and Ferdinandusse contributed equally to this article.

N Engl J Med 2014;371:1900-7. DOI: 10.1056/NEJMoal407778

Copyright @ 2014 Massachusetts Medical Society.
CETOACETATE AND 3-HYDROXYBUTYRATE ARE SLIGHTLY ACIDIC BIOMOLecules that, together with acetone, are called ketone bodies and serve as the major circulating energy source during fasting. Ketone bodies are formed in the liver from the ultimate breakdown product of fatty acids - acetyl coenzyme A (CoA) - by coupling of two acetyl units in a three-step enzymatic process called ketogenesis. Ketone bodies are believed to undergo passive distribution to metabolically active tissues, where they are used as an energy source. ${ }^{1}$

Ketoacidosis, a pathologic state, occurs when ketone formation exceeds ketone utilization. The clinical consequences of ketoacidosis are exemplified by diabetic ketoacidosis, a condition that is marked by vomiting, osmotic diuresis, dehydration, and Kussmaul breathing and that may progress to decreased consciousness and, ultimately, death. ${ }^{2}$

Inborn errors of ketone utilization are manifested similarly; however, glucose levels in these types of ketoacidosis are normal or even low, in contrast to glucose levels in diabetic ketoacidosis. ${ }^{3}$ Only two genetic causes of recurrent ketoacidosis are currently known: succinyl CoA oxoacid transferase (SCOT) deficiency (Online Mendelian Inheritance in Man [OMIM] database number, 245050) and mitochondrial acetoacetyl-CoA thiolase (ACAT1) deficiency (also called beta-ketothiolase deficiency; OMIM number, 203750). Both SCOT and ACAT1 are involved in ketolysis, the breakdown of ketone bodies into the key cellular energy source, acetyl CoA. 
We performed targeted exome sequencing of homozygous genomic regions in a patient of consanguineous descent who had recurrent, severe ketoacidosis. A homozygous mutation was detected in the gene encoding monocarboxylate transporter 1 (MCT1). Subsequently, we evaluated a series of 96 patients with recurrent ketoacidosis, in whom known ketolytic defects had been ruled out enzymatically, to identify additional patients with mutations in MCT1 or related genes.

\section{METHODS}

\section{STUDY PARTICIPANTS AND GENETIC STUDIES}

We performed targeted exome sequencing of homozygous genomic regions in the index patient and her family members. Regions of homozygosity in the index patient were determined with the use of a high-resolution single-nucleotide polymorphism (SNP) array. Coding parts of homozygous regions were then captured on a custom array and sequenced as described elsewhere. ${ }^{4,5}$ To follow up on the findings from exome sequencing, we sequenced the entire coding region of MCT1 in a series of 96 patients with ketoacidosis in whom known ketolytic defects had been ruled out because of the normal enzymatic activities of ACAT1 and SCOT. In addition, we performed Sanger sequencing of the related genes MCT2 (SLC16A7), MCT3 (SLC16A8), and MCT4 (SLC16A3), plus the ancillary gene BSG, in these patients. Heterozygosity of mutations was confirmed by means of complementary DNA (cDNA) sequencing and genomic deletion analysis.

Further details of sequencing and analysis are available in the Supplementary Appendix, available with the full text of this article at NEJM.org. Written informed consent for targeted wholeexome sequencing and Sanger sequencing was obtained from the parents of the index patient for themselves, the index patient, and her two siblings. Sanger sequencing of candidate genes in the cohort of patients with a suspected ketolytic defect and blood collection for functional studies were performed as a part of the diagnostic process (approved by the medical ethics committee of the University Medical Center Utrecht).

\section{MCTI EXPRESSION AND FUNCTIONAL STUDIES}

Erythrocyte lactate transport was measured essentially as described by Fishbein. ${ }^{6}$ For the analysis of each blood sample from a patient, at least one control sample was included in the same ex- periment, with the same procedures used for blood collection, transport, and analysis. Control blood samples were taken at random from blood left over from diagnostic tests, which was used anonymously in this study. Immunoblotting was performed with fibroblast homogenates and an affinity-purified rabbit polyclonal antibody against MCT1 and MCT4. Fibroblast homogenates from 10 healthy volunteers were also used as controls. Further details on functional and expression studies are available in the Supplementary Appendix. Reference blood results from testing of children before and after 24 hours of fasting were obtained from published data.

\section{STATISTICAL ANALYSIS}

All applied statistical tests were two-tailed unpaired t-tests. P values of less than 0.05 were considered to indicate statistical significance.

\section{RESULTS}

\section{CASE REPORT}

The index patient had repetitive episodes of profound metabolic acidosis, with a blood pH below 7.00 on three occasions, all accompanied by massive urinary excretion of 3-hydroxybutyrate and acetoacetate, with plasma lactate and ammonia levels remaining in the normal range. Between episodes, results of blood gas analyses were normal. See the Supplementary Appendix for an extended case report.

\section{GENETIC STUDiEs}

SNP array analysis in the index patient revealed 36 homozygous genomic regions larger than $1 \mathrm{Mb}$, with 7 of the 36 larger than $10 \mathrm{Mb}$, which confirmed consanguinity. Targeted exome sequencing of coding parts of these homozygous regions in the five family members yielded nine rare variants. Of these, a single-nucleotide insertion disrupting the reading frame of MCT1 at asparagine 15 (c.41dupC; National Center for Biotechnology Information reference sequence number, NM_001166496.1) was the strongest candidate. The correct segregation of this mutation in the family was confirmed by means of Sanger sequencing. This variant was absent from multiple large variation databases (1000 Genomes, dbSNP, and the National Heart, Lung, and Blood Institute [NHLBI] GO Exome Sequencing Project Exome Variant Server [data release ESP6500]). The insertion of an extra nucleotide early in the gene se- 
quence results in a frameshift and thus in a loss of MCT1 function.

\section{MCTI MUTATIONS IN ADDITIONAL PATIENTS WITH KETOACIDOSIS}

MCT1 is one of the transmembrane transporters encoded by members of the SLC16 gene family; among these transmembrane transporters, MCT1, MCT2, MCT3, and MCT4 have been shown to transport monocarboxylates, including lactate and ketone bodies. ${ }^{8}$ MCT1 requires the glycoprotein BSG (also called CD147) for proper subcellular expression. ${ }^{9,10}$ We therefore sequenced MCT1 and related genes in a series of 96 patients with unexplained ketoacidosis. We identified seven additional suspected deleterious variants, all in MCT1, including six truncating mutations and one missense mutation (Fig. 1A and Table 1). All truncating mutations are expected to result in a loss of protein function. The missense mutation, p.Arg313Gln, targets a highly conserved residue that is essential for catalytic activity. ${ }^{11}$ In total, we identified 9 patients with mutations in MCT1, 3 homozygous and 6 heterozygous, including 2 siblings. None of these mutations were seen previously in population-based variation databases (1000 Genomes, dbSNP, NHLBI GO Genome Sequencing Project Exome Variant Server, and Genome of the Netherlands). ${ }^{12}$ Together, the genetic data firmly link defects in MCT1 to ketoacidosis in our patients.

\section{MCTI EXPRESSION}

Next, we studied the effect of the mutations in MCT1 at the protein level. Immunoblot analysis with fibroblast homogenates from patients with a homozygous MCT1 mutation, performed with antibodies raised against a C-terminal peptide of MCT1, confirmed that predicted truncating mutations lead to an absence of the full-length protein (Fig. S1A in the Supplementary Appendix). In five patients, we could detect only a single heterozygous truncating mutation in MCT1 (Fig. 1A), despite sequencing the complete coding region. Analysis of cDNA confirmed biallelic expression (Fig. 1B). A custom multiplex amplification quantification analysis further ruled out the presence of exon-sized deletions in heterozygous patients. Immunoblot analysis showed significantly reduced levels of MCT1 protein in fibroblasts from patients with a heterozygous truncating mutation, relative to levels in controls (Fig. 1C, and Fig S1B in the
Supplementary Appendix). The immunoblot results confirmed that there was an MCT1 deficiency at the protein level. We tested fibroblasts to determine whether the MCT1 deficiency was compensated for by increased cellular expression of MCT4 but found no evidence for this (Fig S1C in the Supplementary Appendix).

\section{MONOCARBOXYLATE TRANSPORT ASSAY}

Next, we studied the effect of mutations in MCT1 on monocarboxylate transport, using a previously described assay for MCT1 activity that measures lactate efflux from erythrocytes endogenously loaded with lactate. ${ }^{6,13}$ In the samples from patients with a homozygous mutation in MCT1, erythrocyte lactate transport was significantly reduced as compared with that in the control samples (Fig. 1D). The mean lactate transport activity in erythrocytes from heterozygous carriers, both symptomatic and asymptomatic, was significantly reduced as compared with the transport activity in control samples but was significantly higher than that in homozygous patients (Fig. 1D).

\section{CLINICAL SYMPTOMS}

All patients presented with bouts of ketoacidosis provoked by fasting or infections in their first years of life (Table 1), as is illustrated for the index patient in Figure 2A, and Figure S2A in the Supplementary Appendix. The $\mathrm{pH}$ of the blood was normal between episodes. Excretion of urinary ketones under these circumstances ranged from normal to clearly elevated (Fig. 2B). Ketoacidotic episodes were preceded by poor feeding and vomiting and were associated with dehydration, which was a consequence of osmotic diuresis and vomiting. Hypoglycemia was observed infrequently, and glucose levels usually remained in the normal range (Fig. S2B and S2C in the Supplementary Appendix). In some patients, repetitive vomiting led to a diagnosis of ketotic vomiting or abdominal migraine. Profound metabolic acidosis was associated with decreased consciousness and insufficient respiratory drive (Fig. 2C), which worsened the degree of acidosis.

In all patients, treatment with intravenous glucose or dextrose (combined with bicarbonate) led to rapid clearance of metabolic acidosis. Early initiation of treatment appeared to prevent ketoacidosis. Similarly, ensuring an adequate caloric intake reduced the number of episodes. The frequency of ketoacidotic episodes appeared to de- 


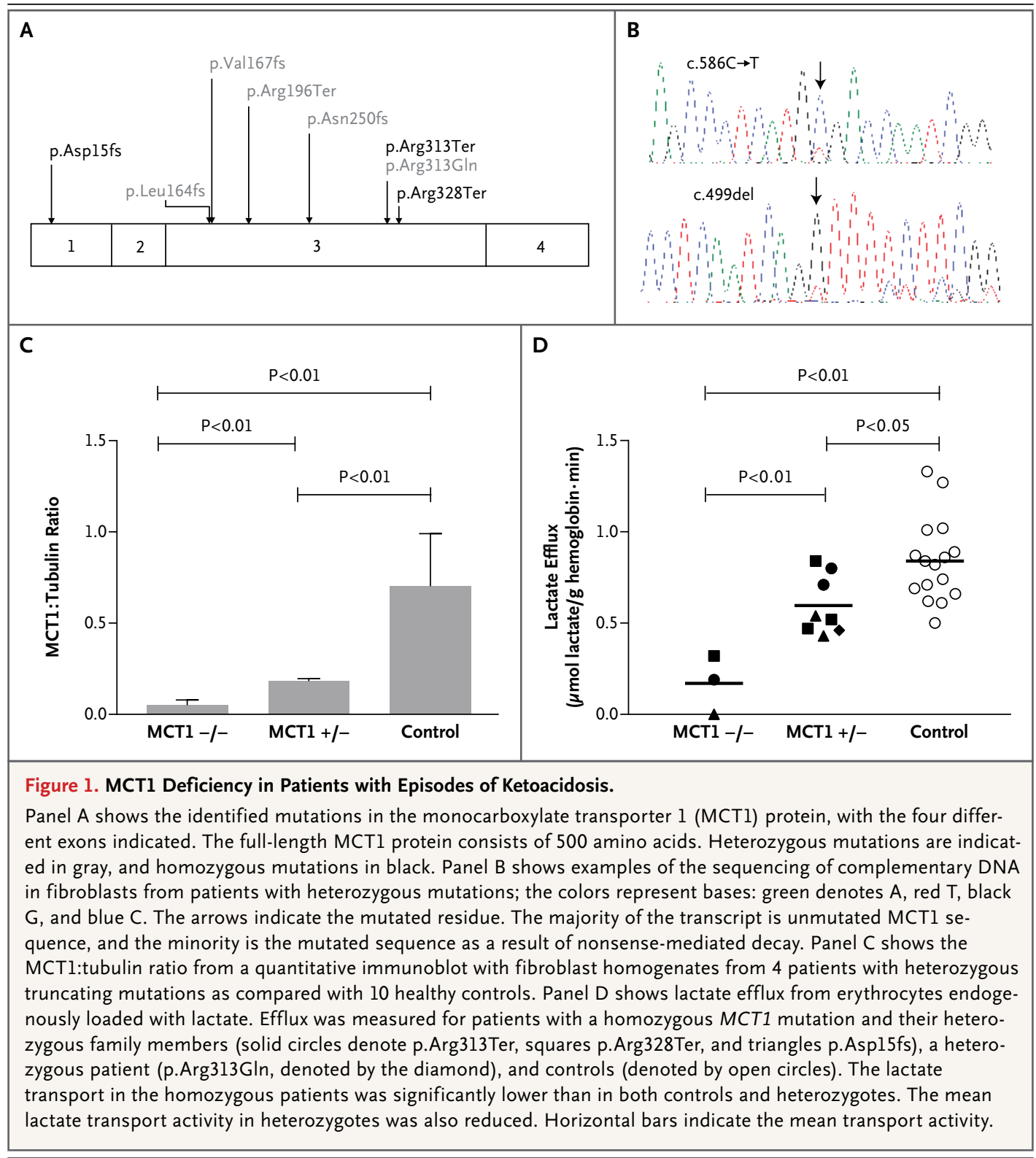

crease over time, and none of the patients had documented ketoacidosis after 7 years of age. Nevertheless, some patients continued to have marked ketonuria associated with (mild) infections.

Patients with homozygous mutations in MCT1 tended to present at a younger age $(\mathrm{P}=0.05)$ and had more profound ketoacidosis (Fig. 2D, and Fig. S2D in the Supplementary Appendix). In addition, homozygous patients had mild-tomoderate developmental delay, whereas patients with milder deficiencies of MCT1 had normal development.

\section{DISCUSSION}

We identified MCT1 deficiency as a disorder of ketone utilization, expanding the spectrum of disorders leading to ketoacidosis from those of ketolysis to those of ketone delivery. This finding may aid in timely diagnosis of the disorder and allow for improved disease management. In addition, this observation implies that MCT1mediated import of ketone bodies into extrahepatic tissues is essential during periods of catabolic stress in order to maintain acid-base balance. 


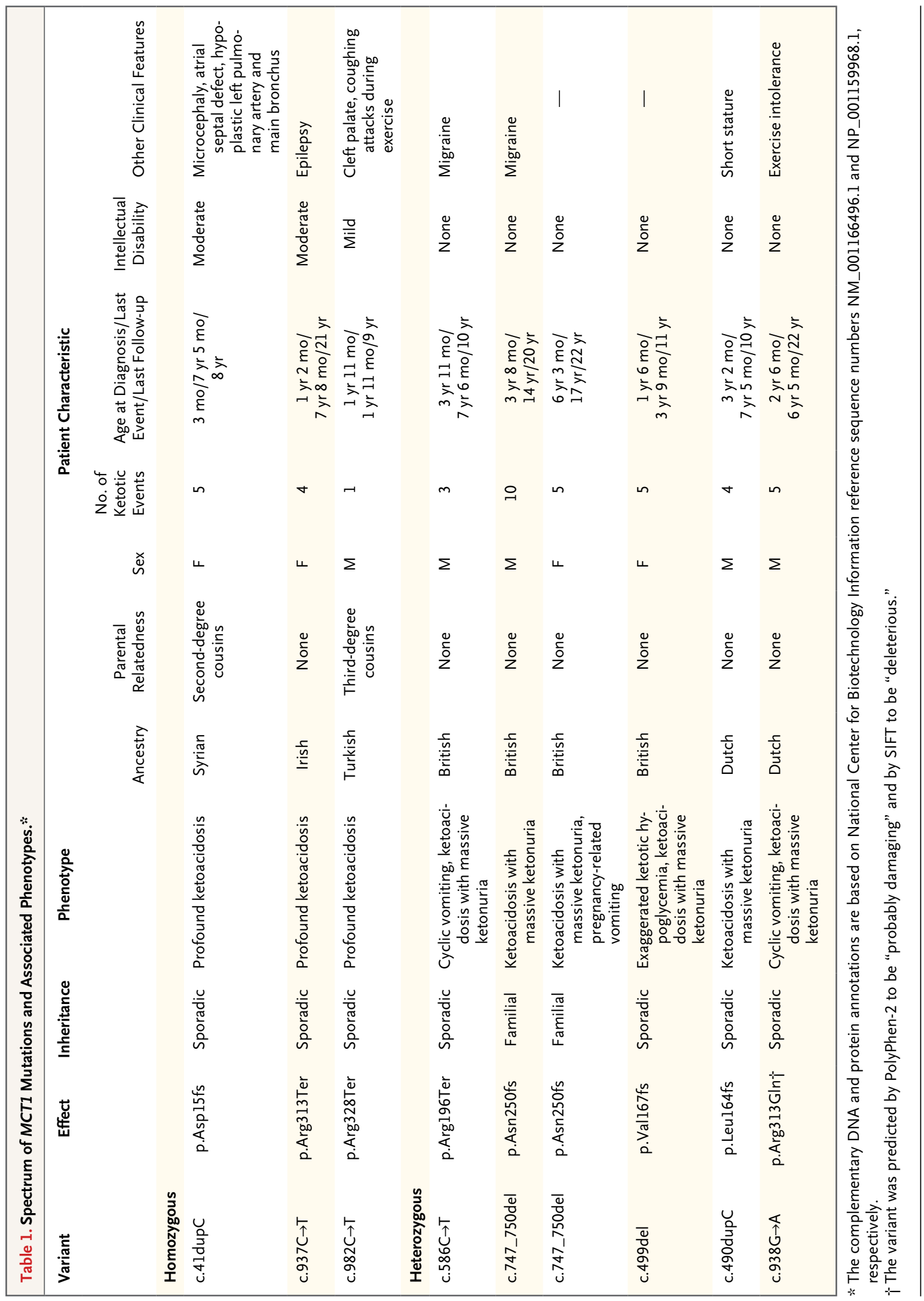




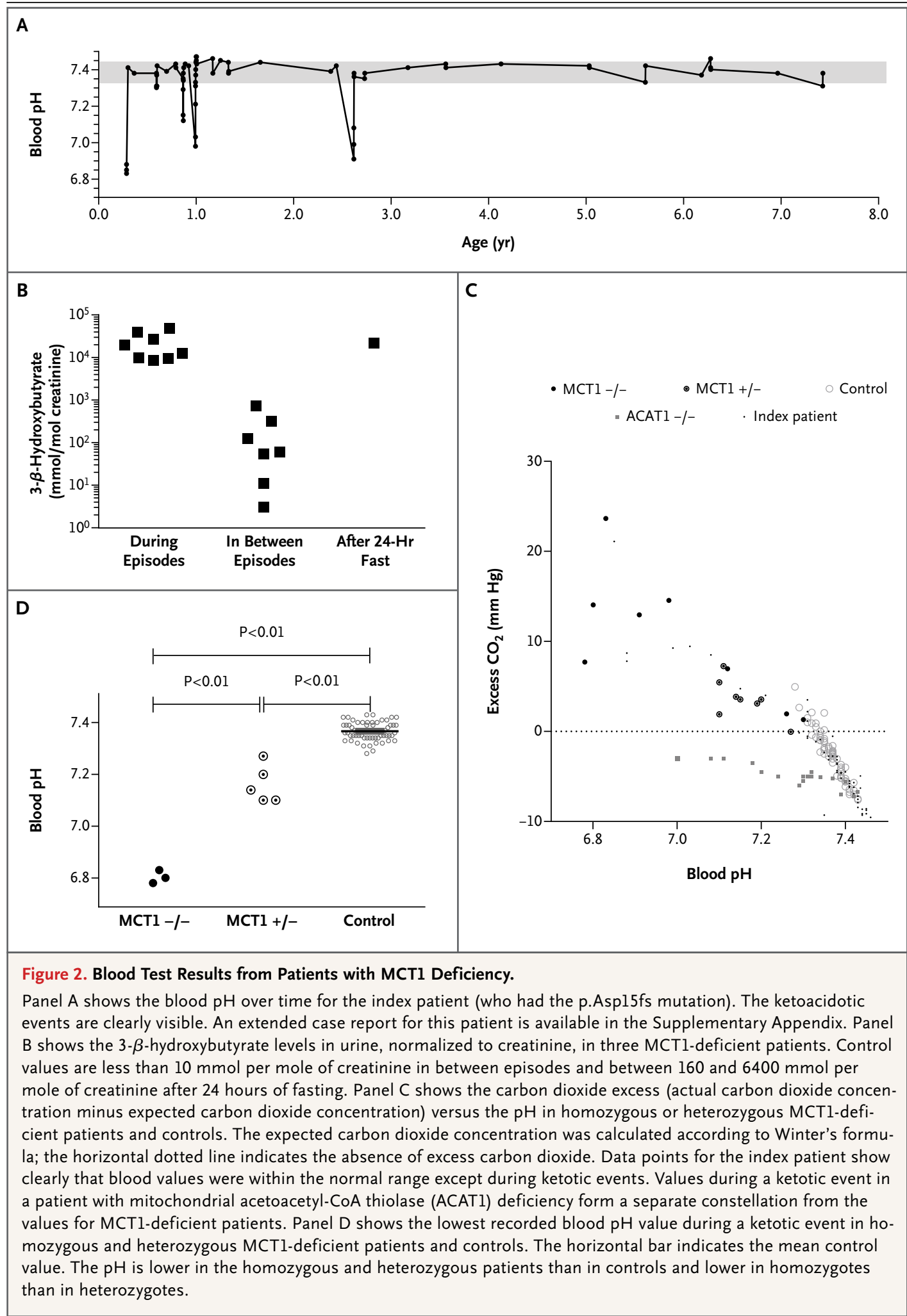

N ENGLJ MED 371;20 NEJM.ORG NOVEMBER 13, 2014

The New England Journal of Medicine 
MCT1 encodes a transmembrane protein that facilitates proton-linked transport of a range of monocarboxylate metabolites, such as lactate, pyruvate, and ketone bodies, across the cellular membrane. ${ }^{8}$ Our observation of profound ketoacidosis in patients with MCT1 deficiency highlights the fact that facilitated transport of ketone bodies by MCT1 is essential during catabolic stress, when ketone turnover in the body is orders of magnitude higher than in the fed state.

Heterozygous missense mutations in MCT1 have previously been reported in association with suboptimal erythrocyte lactate transport resulting in muscle injury on exercise and heat exposure. ${ }^{13}$ Our patient with a heterozygous missense mutation had exercise intolerance, which indicates a possible overlapping phenotype. Mutations leading to increased expression of MCT1 are associated with exercise-induced hyperinsulinism. ${ }^{14,15}$

Monocarboxylate transporters show tissuespecific variation in expression between species, ${ }^{16}$ which underlines the importance of insights gained in our study of MCT1 function in human disease. Homozygous MCT1-knockout mice die early during embryogenesis, ${ }^{17,18}$ in contrast to the patients with a complete loss of MCT1 function in this study. Mouse placenta and human placenta express MCT1 in opposite subcellular arrangements ${ }_{19}^{19}$ which provides a possible explanation for the differences in embryonic lethality. Disruption of MCT1 in the central nervous system produces axon damage and neuronal loss in mice. ${ }^{17}$ The patients with homozygous loss-offunction mutations in MCT1 described in our study have moderate intellectual disability; however, at this stage it remains unclear whether this is a direct effect of the absence of MCT1 in the brain or caused by episodes of profound ketoacidosis.
The use of acidic biomolecules as an energy source presents a potential threat to acid-base homeostasis and therefore requires a careful balance between production and consumption. This aim is achieved indirectly, by linking the rate of ketogenesis with cellular energy status in extrahepatic tissues. Concerted action of glucagon and insulin released by the pancreas determines the rate of ketogenesis in the liver. The massive ketoacidosis in patients with MCT1 deficiency and other disorders of ketone utilization - SCOT deficiency and ACAT1 deficiency - unveils the weak spot of this indirect feedback mechanism. In these disorders, the conversion of ketone bodies to acetyl CoA becomes rate limiting. As a consequence, ketone production is uncoupled from consumption, leading to profound metabolic acidosis.

We found that a single heterozygous MCT1 mutation can result in a deficiency of the transporter and in clinical symptoms. These findings suggest that MCT1 deficiency is more prevalent than is apparent among previously described disorders of ketone utilization. The heterozygous family members of patients with homozygous MCT1 loss-of-function mutations had no history of ketoacidosis, which strongly suggests that heterozygous mutations - and possibly even homozygous mutations - in MCT1 give rise to ketoacidosis only in conjunction with certain genetic and environmental factors. The varying number of episodes and long symptom-free intervals in both heterozygous and homozygous patients support this hypothesis. Future research will have to identify the factors involved in triggering the development of symptoms.

Supported by the Child Health program of the University Medical Center Utrecht.

Disclosure forms provided by the authors are available with the full text of this article at NEJM.org.

We thank Aafke Terlingen for technical assistance.

\section{APPENDIX}

The authors' affiliations are as follows: the Division of Pediatrics, Department of Metabolic Diseases (P.M.H., G.V.), and the Division of Pediatrics, Department of Pediatric Gastroenterology (R.H.J.H.), Wilhelmina Children's Hospital, and the Center for Molecular Medicine, Department of Medical Genetics (G.R.M., M.J.G., K.D., M.H., B.Z., J.J.S., N.M.V.-D., G.H.), University Medical Center Utrecht, Utrecht, Laboratory Genetic Metabolic Diseases, Departments of Clinical Chemistry and Pediatrics, Academic Medical Center, Amsterdam (S.F., J.P.N.R., M.T., R.J.A.W.), the Division of Pediatrics, Department of Metabolic Diseases, and Laboratory Genetic Metabolic Diseases, Maastricht University Medical Center, Maastricht (M.E.R.-G.), and the Department of Pediatrics, Nijmegen Center for Mitochondrial Disorders, Radboud University Medical Center, Nijmegen (M.C.V.) — all in the Netherlands; the National Centre for Inherited Metabolic Disorders, Children's University Hospital, Dublin, Ireland (A.A.M.); the Department of Pediatric Metabolism and Nutrition, Gazi University School of Medicine, Ankara, Turkey (I.O.); and the Department of Paediatric Metabolic Medicine, Sheffield Children's Hospital, Sheffield (M.J.S.), the Department of Metabolic Medicine, Great Ormond Street Hospital NHS Foundation Trust, London (M.C.), Chemical Pathology, Department of Laboratory Medicine, Salisbury (N.O.), and the Department of Clinical Biochemistry, Southampton General Hospital, Southampton (V.W.) - all in the United Kingdom. 
REFERENCES

1. Laffel L. Ketone bodies: a review of physiology, pathophysiology and application of monitoring to diabetes. Diabetes Metab Res Rev 1999;15:412-26.

2. Westerberg DP. Diabetic ketoacidosis evaluation and treatment. Am Fam Physician 2013;87:337-46.

3. Sass JO. Inborn errors of ketogenesis and ketone body utilization. J Inherit Metab Dis 2012;35:23-8

4. Nijman IJ, Mokry M, van Boxtel R, Toonen P, de Bruijn E, Cuppen E. Mutation discovery by targeted genomic enrichment of multiplexed barcoded samples. Nat Methods 2010;7:913-5.

5. Harakalova M, Mokry M, Hrdlickova $\mathrm{B}$, et al. Multiplexed array-based and insolution genomic enrichment for flexible and cost-effective targeted next-generation sequencing. Nat Protoc 2011;6:1870 86.

6. Fishbein WN. Lactate transporter defect: a new disease of muscle. Science 1986;234:1254-6.

7. van Veen MR, van Hasselt PM, de Sain-van der Velden MG, et al. Metabolic profiles in children during fasting. Pediatrics 2011;127(4):e1021-7.

8. Halestrap AP. The SLC16 gene family - structure, role and regulation in health and disease. Mol Aspects Med 2013;34 337-49.
9. Kirk P, Wilson MC, Heddle C, Brown MH, Barclay AN, Halestrap AP. CD147 is tightly associated with lactate transporters MCT1 and MCT4 and facilitates their cell surface expression. EMBO J 2000;19: 3896-904.

10. Wilson MC, Meredith D, Fox JE, Manoharan C, Davies AJ, Halestrap AP. Basigin (CD147) is the target for organomercurial inhibition of monocarboxylate transporter isoforms 1 and 4: the ancillary protein for the insensitive MCT2 is EMBIGIN (gp70). J Biol Chem 2005;280: 27213-21.

11. Manoharan C, Wilson MC, Sessions $\mathrm{RB}$, Halestrap AP. The role of charged residues in the transmembrane helices of monocarboxylate transporter 1 and its ancillary protein basigin in determining plasma membrane expression and catalytic activity. Mol Membr Biol 2006;23: 486-98.

12. Genome of the Netherlands Consortium. Whole-genome sequence variation, population structure and demographic history of the Dutch population. Nat Genet 2014;46:818-25.

13. Merezhinskaya N, Fishbein WN, Davis JI, Foellmer JW. Mutations in MCT1 cDNA in patients with symptomatic deficiency in lactate transport. Muscle Nerve 2000;23:90-7.
14. Otonkoski T, Jiao H, Kaminen-Ahola $\mathrm{N}$, et al. Physical exercise-induced hypoglycemia caused by failed silencing of monocarboxylate transporter 1 in pancreatic beta cells. Am J Hum Genet 2007;81: 467-74.

15. Marquard J, Welters A, Buschmann $\mathrm{T}$, et al. Association of exercise-induced hyperinsulinaemic hypoglycaemia with MCT1-expressing insulinoma. Diabetologia 2013;56:31-5.

16. Merezhinskaya N, Fishbein WN. Monocarboxylate transporters: past, present, and future. Histol Histopathol 2009;24:243-64.

17. Lee Y, Morrison BM, Li Y, et al. Oligodendroglia metabolically support axons and contribute to neurodegeneration. $\mathrm{Na}$ ture 2012;487:443-8.

18. Lengacher S, Nehiri-Sitayeb T, Steiner $\mathrm{N}$, et al. Resistance to diet-induced obesity and associated metabolic perturbations in haploinsufficient monocarboxylate transporter 1 mice. PLoS One 2013;8(12): e82505.

19. Nagai A, Takebe K, Nio-Kobayashi J, Takahashi-Iwanaga H, Iwanaga T. Cellular expression of the monocarboxylate transporter (MCT) family in the placenta of mice. Placenta 2010;31:126-33.

Copyright (c) 2014 Massachusetts Medical Society.

RECEIVE THE JOURNAL'S TABLE OF CONTENTS EACH WEEK BY E-MAIL

To receive the table of contents of the Journal by e-mail every Wednesday evening, sign up at NEJM.org. 